\title{
Improving the spatial solution of electrocardiographic imaging: A new regularization parameter choice technique for the Tikhonov method
}

\author{
Judit Chamorro-Servent ${ }^{1,2,3,{ }^{*}}$ (0000-0002-5722-0499), Rémi Dubois ${ }^{1,4,5}$, \\ Mark Potse $^{1,2,3}$ (0000-0003-4166-2687), Yves Coudière ${ }^{1,2,3}$ \\ ${ }^{1}$ IHU Liryc, Electrophysiology and Heart Modeling Institute, foundation Bordeaux \\ Université, Pessac-Bordeaux, France \\ ${ }^{2}$ CARMEN Research Team, INRIA, Bordeaux, France \\ ${ }^{3}$ Univ. Bordeaux, IMB, UMR 5251, CNRS, INP-Bordeaux, Talence, France \\ ${ }^{4}$ Univ. Bordeaux, CRCTB, U1045, Bordeaux, France \\ ${ }^{5}$ INSERM, CRCTB, U1045, Bordeaux, France \\ judit.chamorro-servent@inria.fr
}

\begin{abstract}
The electrocardiographic imaging (ECGI) inverse problem is highly ill-posed and regularization is needed to stabilize the problem and to provide a unique solution. When Tikhonov regularization is used, choosing the regularization parameter is a challenging problem. Mathematically, a suitable value for this parameter needs to fulfill the Discrete Picard Condition (DPC). In this study, we propose two new methods to choose the regularization parameter for ECGI with the Tikhonov method: i) a new automatic technique based on the DPC, which we named ADPC, and ii) the U-curve method, introduced in other fields for cases where the well-known L-curve method fails or provides an over-regularized solution, and not tested yet in ECGI. We calculated the Tikhonov solution with the ADPC and U-curve parameters for in-silico data, and we compared them with the solution obtained with other automatic regularization choice methods widely used for the ECGI problem (CRESO and L-curve). ADPC provided a better correlation coefficient of the potentials in time and of the activation time (AT) maps, while less error was present in most of the cases compared to the other methods. Furthermore, we found that for in-silico spiral wave data, the L-curve method over-regularized the solution and the AT maps could not be solved for some of these cases. U-curve and ADPC provided the best solutions in these last cases.
\end{abstract}

Keywords: inverse problem; regularization; electrocardiographic imaging; potentials; Tikhonov regularization; ill-posed problems. 


\section{Introduction}

During the past decades, much progress has been made in solving the inverse problem of electrocardiography [1-7]. However, despite all the success of non-invasive electrocardiographic imaging (ECGI), the understanding and treatment of many cardiac diseases is not feasible yet without an improvement of the inverse problem solution [8]. Solution of the 'inverse problem' depends on the 'forward problem', i.e. on specification of the relationship between potential sources on the cardiac surface, $\Phi_{E}$, and the body surface measured potentials, $\Phi_{T}$.

The inverse problem (finding $\Phi=\Phi_{\mathrm{E}}$ at epicardial surface $\Gamma_{E}$ ) is innately ill-posed. That means that is extremely sensible to: i) noise on the measured potentials, ii) uncertainty in the location of measurement sites with respect to the surface on which the sources are distributed, iii) errors of segmentation of the geometries, and iv) influence of cardiac motion.

Regularization incorporates additional knowledge in the inverse problem by applying constraints to the solution, to stabilize the problem yielding realistic and unique results. When regularization is applied, the weight of the constraints (regularization parameter) has to be determined to find a balance between solutions purely based on the body-surface potentials and solutions that are constrained too strictly. While the former may be severely distorted by ill-posedness, the latter may have too much bias. Given the ill-posedness, the regularization parameter choice has an important influence on the solution [8].

In this study we focused on the two-norm Tikhonov regularization technique for the method of fundamental solution (MFS), an homogeneous meshless method adapted to ECGI by Wang and Rudy $[9,10]$. Specifically, we will focus on the choice of the regularization parameter. In many implementations, the Tikhonov regularization problem is solved by manually selecting the regularization parameter $\alpha$. This is done using a sequence of regularization parameters and selecting the value that gives the best results, as judged by the user. Obviously, the procedure is subjective and time consuming. To overcome this problem, several automatic methods for selecting regularization parameters have been suggested over the years. These include: i) Strategies based on the calculation of the variance of the solution, which requires prior knowledge of the noise, ii) Strategies that do not need a priori information. For ECGI, we are interested in the latter.

Regarding the second group (ii) of automatic methods previously used in the MFS ECGI literature [9,10], the regularization parameter is obtained by using the mean of the regularization parameters provided by the Composite Residual and Smoothing Operator (CRESO) technique [10]. The CRESO method has been found to perform comparably to the "optimal" regularization parameter that provides the minimum root-meansquare error (RMSE) between the computed epicardial potentials $\left(\Phi_{E}\right)$ and the measured ones [10]. When other numerical methods, such as the Boundary Element method (BEM), were used to solve the ECGI problem, the L-curve criterion has been highly used by the community to find the regularization parameter $[8,11]$.

While the efficacy of the CRESO and L-curve methods has been proven in the wide inverse problems literature [8-16], finding an automatic regularization parameter for 
Tikhonov regularization that is suitable for all ill-posed inverse problems is a challenging problem [12, 15] and CRESO and L-curve may over-regularize the solution or fail for some cases. This is especially important when we deal with medical problems such as cardiac pathologies, where different pathologies may need different regularization.

For this reason, the overarching goal of this paper is to show the feasibility of the Ucurve method [17-18], not used yet in cardiac inverse problems, after our knowledge; and to develop a new automatic method, ADPC, based on the Discrete Picard Condition (DPC), a mathematical condition that any suitable regularization parameter for Tikhonov regularization must fulfill [12].

\section{Methods}

\subsection{Method of fundamental solutions and Tikhonov regularization}

MFS is a homogeneous meshless approach that was adapted to ECGI to overcome some of the issues of the classical meshes-based methods [9-10]. MFS does not need the topological relations between nodes and so completely avoids disadvantages of accuracy degradation and complexity augmentation frequently encountered in classical numerical methods because of remeshing. Thus it avoids, negative effects introduced by errors in the segmentation process and/or singularities in the boundaries.

In the MFS, the potentials are expressed as a linear combination of Laplace fundamental solution over a discrete set of virtual source points (Dirac masses) placed outside of the domain of interest, $\Omega$, where $\Omega$ is the volume conductor enclosed by the epicardial surface $\Gamma_{E}$ and the body surface, $\Gamma_{T}$. Specifically the potential $\Phi$ for $x \in \Omega$ is sought as

$$
\Phi(x)=a_{0}+\sum_{j=1}^{N_{S}} f\left(x-y_{j}\right) a_{j},
$$

where the $\left(y_{j}\right)_{j=1 . . N_{S}}$ are the $N_{S}$ locations of the sources $\left(y_{j} \notin \Omega\right)$, and the $\left(a_{j}\right)_{j=1 . . N_{S}}$ are their coefficients. Here, $f$ stands for the fundamental solution to the Laplace equation, $f(r)=\frac{1}{4 \pi} \frac{1}{|r|}$ where $r$ is the 3D Euclidean distance between point $x$ and virtual source $y$. After discretization on collocation points on $\Gamma_{T}$, and fixed number $N_{S}$ of source locations [9], this formulation yields a linear system of equations with matrix system $M$ :

$$
M=\left(\begin{array}{cccc}
1 & f\left(r_{11}\right) & \cdots & f\left(r_{1 N_{S}}\right) \\
\vdots & & \ddots & \vdots \\
1 & f\left(r_{N_{T} 1}\right) & \cdots & f\left(r_{N_{T} N_{S}}\right) \\
0 & \partial_{n_{1}} f\left(r_{11}\right) & \cdots & \partial_{n_{1}} f\left(r_{1 N_{S}}\right) \\
\vdots & & \ddots & \vdots \\
0 & \partial_{n_{N_{T}}} f\left(r_{N_{T} 1}\right) & \cdots & \partial_{n_{N_{T}}} f\left(r_{N_{T} N_{S}}\right)
\end{array}\right),
$$

where $r_{i j}=x_{i}-y_{j}, x_{i} \in \Gamma_{T}$ and $\left(y_{j}\right)_{j=1 . . N_{S}}, y_{j} \notin \Omega$. Hence, homogeneous Neumann conditions (no flux conditions, $\partial_{n} \Phi=0$ ) and Dirichlet conditions ( $\Phi=\Phi_{T}$, where 
$\Phi_{T}$ are the potentials acquired on the torso) on $\Gamma_{T}$ are considered in an apparently equivalent manner in the standard MFS described in [9]. Therefore, only the sources coefficients are unknown, resulting in a quadratic minimizing problem (Tikhonov regularization problem), find the sources coefficients $a=\left(a_{0}, a_{1}, \cdots, a_{N_{S}}\right)^{T} \in \mathbb{R}^{1+N_{S}}$ that minimize

$$
J(a, \alpha)=\|\mathrm{M} a-\mathrm{b}\|^{2}+\alpha\|a\|^{2}
$$

where $b=\left(\begin{array}{c}\Phi_{T}^{*} \\ 0\end{array}\right)$ is a $2 N_{T} x 1$ vector, given some potentials $\Phi_{T}^{*}=\left(\Phi_{i}^{*}\right)_{i=1, \cdots, N_{T}}$ recorded on $N_{T}$ torso electrodes, and $\alpha>0$ is the Tikhonov regularization parameter. Note that the first term $\|\mathrm{M} a-\mathrm{b}\|^{2}$ measures the goodness-of-fit, i.e., how well the unknown sources coefficients $a$ predicts the given boundary conditions at body surface. The second term $\|a\|^{2}$ measures the regularity of the sources coefficients $a$. It suppresses (most of) its large noise components by controlling its norm (since the naïve solution is dominated by high-frequency components with large amplitudes). The balance between both terms is controlled by the regularization parameter, $\alpha$.

Once solved (2) and found the source coefficients $a=\left(a_{0}, a_{1}, \cdots, a_{N_{S}}\right)^{T} \in \mathbb{R}^{1+N_{s}}$, the $\Phi_{\mathrm{E}}$ are found by using (1) for $x \in \Gamma_{E}$ and $\left(y_{j}\right)_{i=1 . . N_{S}}, y_{j} \notin \Omega$.

\subsection{Discrete Picard Condition}

Instead of the condition number of the matrix of a linear system, the rate of decrease of the singular values (SVs) is a better indication of the conditioning of the problem [12, 13]. Furthermore, the Discrete Picard Condition (DPC) [12] provides an objective assessment of the ill-posedness of the entire problem relating the matrix information (by means of the SVs) with the measurements [12].

The Tikhonov solution can be obtained by equalling the gradient of (2) to zero:

$\nabla J_{a}(a, \alpha)=\nabla\left((M a-b)^{T}(M a-b)+\alpha I a^{T} a\right)=\left(M^{T} M\right) a-M^{T} b+\alpha^{2} I a=0$, and $a_{\alpha}=\left(M^{T} M+\alpha^{2} I\right)^{-1} M^{T} b$.

Then, by decomposing the forward matrix of the MFS in terms of SV decomposition $\left(M=U S V^{T}\right)$ and writing $I=V V^{T}$,

$$
a_{\alpha}=\sum_{i=1}^{\min \left(2 * N_{T}, N_{S}+1\right)} \frac{\sigma_{i}}{\sigma_{i}^{2}+\alpha^{2}} u_{i}^{T} b v_{i}=\sum_{i=1}^{\min \left(2 * N_{T}, N_{S}+1\right)} \frac{\sigma_{i}^{2}}{\sigma_{i}^{2}+\alpha^{2}} \frac{u_{i}^{T} b}{\sigma_{i}} v_{i}
$$

where $\sigma_{i}$ are the SVs in descending order, $\sigma_{1} \geq \cdots \geq \sigma_{\min \left(2 * N_{T}, N_{S}+1\right)}$ (the elements of the diagonal matrix $S$ ).

The DPC is satisfied if the data space coefficients $\left|u_{i}^{T} b\right|$, on average, decay to zero faster than the respective $\sigma_{i}$ 's. The representation of $\left|u_{i}^{T} b\right|, \sigma_{i}$, and the respective quotient in a same plot in logarithmic scale is known as a Picard plot.

In ill-posed problems, such as ECGI, there may be a point where the data become dominated by errors and the DPC fails. In other words, for larger values of the index $i$ 
the solution coefficients $\frac{\left|u_{i}^{T} b\right|}{\sigma_{i}}$ start to increase and the computed solutions (3) are completely dominated by the SVD coefficients corresponding to the smallest SVs. In these cases, to compute a satisfactory solution by means of Tikhonov regularization, the DPC has to be fulfilled [12,18]. That is, the $\sigma_{i}$ above the regularization parameter $\alpha$ (useful SVs) must decay to zero slower than the corresponding right hand side coefficients, $\left|u_{i}^{T} b\right|$. The DPC determines how well the regularized solution approximates the unknown, exact solution.

\subsection{Existent regularization parameter choice techniques}

\section{Composite Residual and Smoothing Operator (CRESO)}

CRESO was developed by Colli-Franzone et al. [14]. The method was presented as an empirical heuristic but has become widely accepted as the preferred method of parameter estimation in a large class of ill-posed bioelectric inverse problems [15]. The CRESO method chooses that parameter value which generates the first local maximum of the difference between the derivative of the constraint term and the derivative of the residual term

$$
C(\alpha)=\left\{\frac{d}{d\left(\alpha^{2}\right)}\left(\alpha^{2}\|x(\alpha)\|^{2}\right)-\frac{d}{d\left(\alpha^{2}\right)}\|M x(\alpha)-b\|^{2}, \alpha>0\right\}
$$

\section{L-curve}

The best-known method for estimating a value for the regularization parameter is defined in terms of the L-curve $[15,16]$

$$
L(\alpha)=\{(\|M x(\alpha)-b\|,\|x(\alpha)\|), \alpha>0\}
$$

To choose the L-curve regularization parameter we used the criterion proposed by Hansen and O'Leary [16], where the optimal $\alpha$-value corresponds to the point on the loglog plot of the L-curve possessing maximum curvature.

\section{U-curve}

The U-curve method was proposed by Krawczyck-Stando and Rudnicki [17]. The Ucurve is the plot of the sum of the inverse of the regularized solution norm $\|x(\alpha)\|$ and the corresponding residual error norm $\|M x(\alpha)-b\|$, for $\alpha>0$ on a log-log scale

$$
U(\alpha)=\frac{1}{\|M x(\alpha)-b\|^{2}}+\frac{1}{\|x(\alpha)\|^{2}}
$$

The U-curve is a U-shaped function, where the sides of the curve correspond to regularization parameters for which either the solution norm or the residual norm dominates. The optimum regularization value is the value for which the U-curve has a minimum. U-curve is computationally efficient since it provides an a priori interval where to find this minimum [17-18]. Furthermore, while its feasibility has not been tested yet for ECGI, it has been shown to perform well for some numerical and biomedical problems when the L-curve failed or gave an over-regularized solution [17-18]. 


\subsection{ADPC: A new regularization parameter choice method}

As cited before, a suitable regularization parameter $\alpha$ for Tikhonov regularization must fulfill the DPC. This means that the $\sigma_{i}$ above the suitable $\alpha$ must decay to zero slower than the corresponding $\left|u_{i}^{T} b\right|$, in order to prevent the computed Tikhonov solutions (3) from being completely dominated by the SVD coefficients corresponding to the smallest SVs. Therefore, we performed an automatic regularization parameter choice method based on this condition as follows:

1. We calculate the singular value decomposition of the MFS matrix, $M$, to obtain the left singular vectors $\left(u_{i}\right)$ and the singular values $\left(\sigma_{i}\right)$.

2. For each instant of time, $t_{k}(m s)$, we compute $\left|u_{i}^{T} b_{t_{k}}\right|$ and we fit the $\log \left(\left|u_{i}^{T} b_{t_{k}}\right|\right)$ by a polynomial $p\left(i, \log \left(\left|u_{i}^{T} b_{t_{k}}\right|\right)\right)_{t_{k}}$ of degree from 5 to 7 . Therefore, we have: $p_{t_{1}, \cdots,}, p_{t_{t}}$ polynomials, one for each instant $t_{k}, k=1, \cdots, N_{t}$ instants of time.

3. For each $p_{t_{k}}$, we find: $\alpha_{t_{k}}=\sigma_{\max \{i\}}$, such that $\log \left(\sigma_{i}\right) \geq p_{t_{k}}$, with $\sigma_{i}$ in descending order.

4. The ADPC regularization parameter is: $\alpha=\operatorname{median}\left(\alpha_{t_{k}}\right)$.

Note that step 2 and 3 of this algorithm are empirically choosing the lower limit that any suitable Tikhonov regularization parameter can achieve to still fulfill the DPC. Both steps are looking for the last index $i$ before the SVD coefficients (corresponding to the smallest SVs) start to dominate the solution. That means, before $\log \left(\sigma_{i}\right)$ starts to be smaller than $\log \left(\left|u_{i}^{T} b_{t_{k}}\right|\right)$. The fitting of the $\log \left(\left|u_{i}^{T} b_{t_{k}}\right|\right)$ by a polynomial is done to simplify the search for a suitable index $i$. Since the behavior of the SVs of ECGI MFS problem and the fact that ADPC parameter choice is based on the necessary DPC, our algorithm provides a suitable regularization parameter.

\section{$2.5 \quad$ Simulated data}

To test the effect of the new regularization parameter choice method and to compare with previous ones, eight different activation patterns ( 1 single site pacing in the right ventricular (RV) free wall, 1 single site pacing in the left ventricular (LV) lateral endocardial wall, 1 single site pacing in the LV mid wall, 1 single pacing site in the LV lateral epi and 4 single spiral waves) were simulated [19]. Propagating activation was simulated with a monodomain reaction-diffusion model in a realistic 3D model of the human ventricles, with transmembrane ionic currents computed with the Ten Tusscher et al. model for the human ventricular myocyte [20]. The transmembrane currents from the monodomain problem were used to compute the extracellular potential distribution throughout the torso by solving a static bidomain problem in a heterogeneous, anisotropic torso model at $1 \mathrm{~mm}$ resolution [21]. The torso model used had heterogeneous conductivity, with anisotropic skeletal muscle, lungs, and intracavitary blood. The heart model consisted of left and right ventricles, with a $0.2 \mathrm{~mm}$ spatial resolution, and an anisotropic conduction derived from rule-based fiber orientation. Cardiac and thoracic 
anatomy was based on MRI data. The simulations provide the theoretical, in-silico $\Phi_{T}$ and $\Phi_{E}$ every $1 \mathrm{~ms}$.

\subsection{Analysis of the reconstructed potentials and activations time maps: Statistical approach}

Once reconstructed the potentials on the epicardium, correlations coefficients (CC) and root mean square errors (RMSE) [12], and relative errors $\left(R E=\left\|\Phi_{E}-\widetilde{\Phi_{E}}\right\| /\left\|\Phi_{E}\right\|\right.$, where $\Phi_{E}$ are the simulated potentials and $\widetilde{\Phi_{E}}$ the reconstructed ones), were computed through time. Thus, we compared the reconstructed potentials on the epicardium against the simulated ones. Afterwards, we calculated the three quartiles of the resulting CCs, RMSEs and REs, (Median [1st quartile, 3rd quartile]).

Similarly, we calculated the activation time (AT) maps and the respective CCs and REs (Median, [min, max]).

\section{$3 \quad$ Results}

\subsection{Ill-posedness and Discrete Picard Condition}

The DPC condition allows us to investigate the behavior of the SVD coefficients $\left|u_{i}^{T} b\right|$ (of the right hand side) and $\frac{\left|u_{i}^{T} b\right|}{\sigma_{i}}$ (of the solution (3)). A logarithmic plot of these coefficients, together with the respective SVs, $\sigma_{i}$ (also in logarithmic scale), is often referred to as DPC plot [12]. Given $\sigma_{1} \geq \cdots \geq \sigma_{\min \left(2 * N_{T}, N_{S}+1\right)}$, lower indices $i$ correspond to higher SVs and bigger indices correspond to lower SVs.

The DPC plot of figure 2 is an example for an in-silico single spiral wave in a fixed instant of time chosen here randomly around $t_{k}=100 \mathrm{~ms}$. This choice is based on the behavior of the body-surface potentials of figure 1 and in the literature [23].

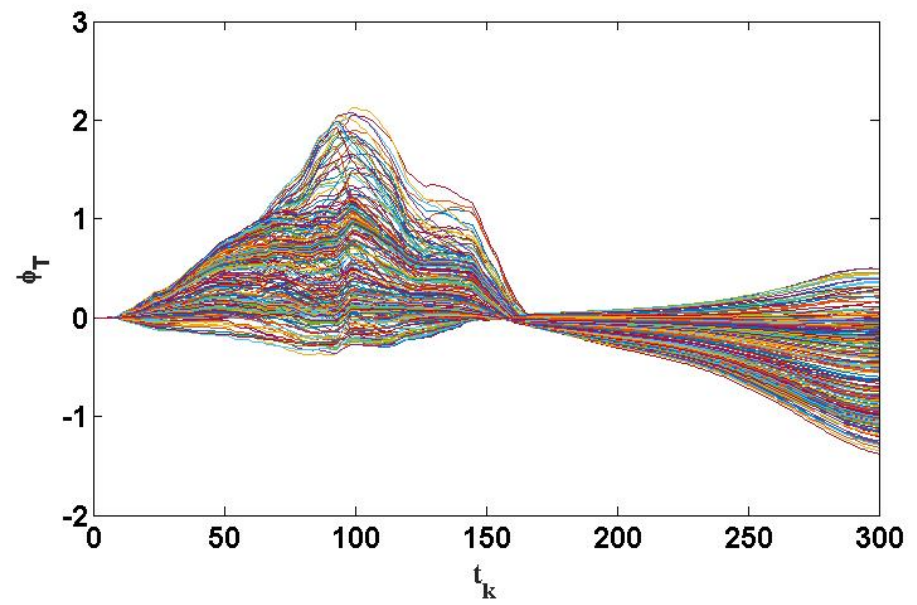

Fig. 1. In-silico body-surface potentials, $\Phi_{T}$ for $t_{k}=1, \cdots, 300 \mathrm{~ms}$ (corresponding to the first beat) of the single spiral wave used for the DPC plot example of figure 2. 


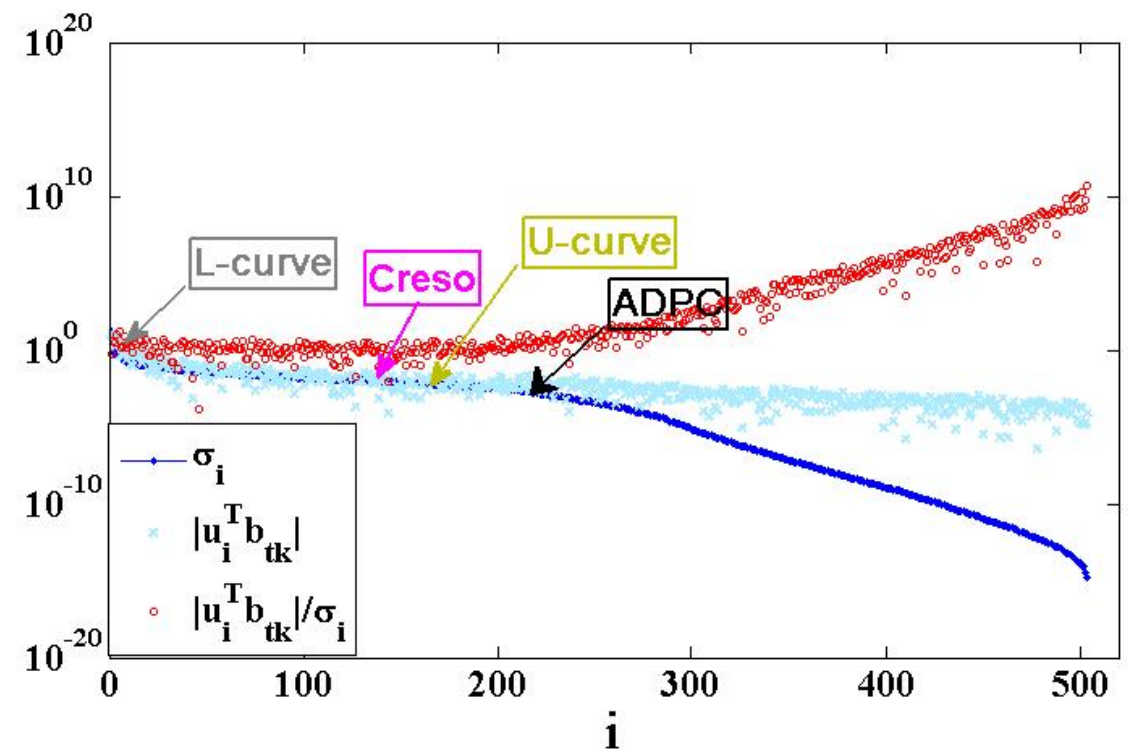

Fig. 2. DPC plot for a time, $t_{k}(m s)$ using the regularization toolbox of Matlab [22] from PC. Hansen The dot points show the decay of $\sigma_{i}$, the crosses $\left|u_{i}^{T} b_{t_{k}}\right|$, and the circles the quotient between both. The values of the different regularization parameter choices are located in the plot with different color arrows and their respective methods' name.

The DPC plot above shows the ill-posedness of the inverse problem. We can see that after the index $i \sim 240 \mathrm{SVs}$ (being $N_{T}=252$ ), the data become dominated by errors/noise and the solution (3) starts to become instable and affected by them. This is due to the fact that for $i>240$, the SVs (dot points), $\sigma_{i}$, start to decrease to zero faster than the right hand side coefficients (crosses) and the DPC fails for any regularization parameter below $\sigma_{240}$. Besides, by holding different color arrows for the different parameter choices on the DPC plot, we can observe the over-regularization provided by the Lcurve method.

We want to remark that while here we only plot the DPC for a certain spiral wave data and for a fixed instant of time, $t_{k}$, (as example to explain the details of a DPC plot) the SVs for the ECGI MFS matrix as formulated in $[9,10]$ usually decrease slowly initially, and they start to highly decrease for larger values of the index $i$ (commonly $i \sim N_{T}$ ). The same SV decay behavior for ECGI MFS for other real torso-heart geometries can be observed in [24].

Figure 3 shows the reconstructed potentials in a random location on the epicardium through the different time instants, $t_{k}$ (ms) for the different regularization parameters together with the simulated ones. We observe that in this example the solution based on the L-curve is over-regularized. 

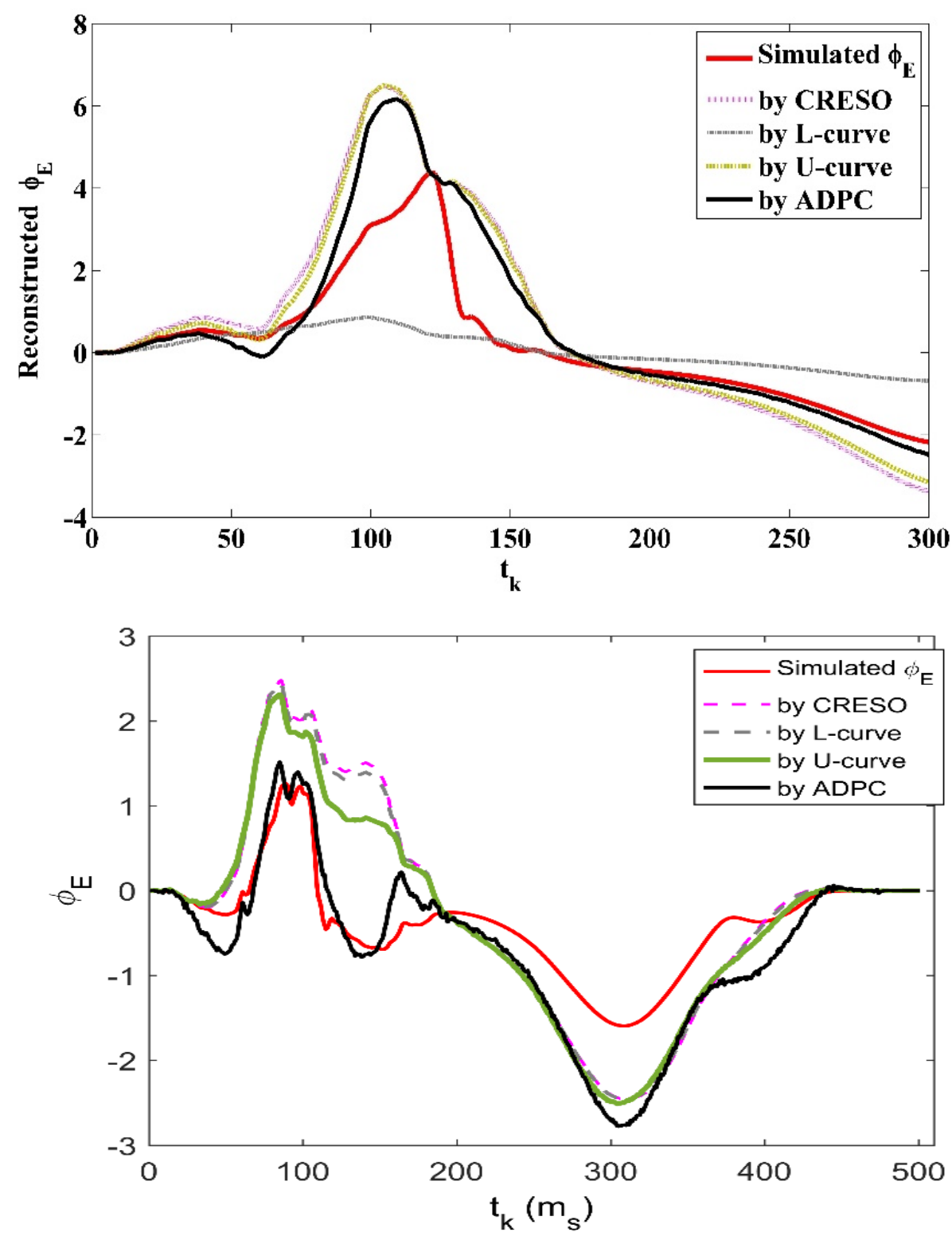

Fig. 3. Signal reconstructed in a point of the epicardium along the time for the different regularization parameters methods as legend indicates against the simulated one. Above: Single site pacing data in the RV, below: single site pacing data in the LV. 


\subsection{Correlation coefficients and relative errors of reconstructed potentials in}

time and activation time maps

Table 1 presents the three quartiles of CCs, RMSEs and REs of the reconstructed potentials with the different regularization parameters found for the single site pacing simulations (SSPs) and single spiral waves simulations (SSWs). Table 2 shows the minimum, median, and maximum of CCs and REs of the respective activation time (AT) maps. In both tables the best CCs, RMSEs and/or REs are outlined in red color.

\begin{tabular}{c|c|c|c|c}
\hline Method & Dataset & CCs & RMSEs & REs (L2-norm) \\
\hline \multirow{2}{*}{ CRESO } & SSPs & $0.77[0.49,0.86]$ & $0.05[0.04,0.06]$ & $0.81[0.62,1.03]$ \\
\cline { 2 - 5 } & SSWs & $0.74[0.57,0.86]$ & $0.07[0.05,0.10]$ & $0.77[0.64,0.96]$ \\
\hline \multirow{2}{*}{-curve } & SSPs & $0.76[0.51,0.85]$ & $0.05[0.04,0.06]$ & $0.89[0.67,1.06]$ \\
\cline { 2 - 5 } & SSWs & $0.70[0.46,0.82]$ & $0.08[0.05,0.13]$ & $0.91[0.80,1.08]$ \\
\hline \multirow{2}{*}{-curve } & SSPs & $0.76[0.53,0.86]$ & $0.06[0.05,0.08]$ & $0.80[0.60,0.97]$ \\
\cline { 2 - 5 } & SSWs & $0.76[0.60,0.87]$ & $0.07[0.05,0.09]$ & $0.75[0.61,0.93]$ \\
\hline \multirow{2}{*}{ ADPC } & SSPs & $0.81[0.70,0.89]$ & $0.06[0.04,0.08]$ & $0.71[0.54,0.83]$ \\
\cline { 2 - 5 } & SSWs & $0.81[0.68,0.90]$ & $0.06[0.04,0.09]$ & $0.69[0.55,0.87]$ \\
\hline
\end{tabular}

Table 1. Median [ $1^{\text {st }}$ quartile, $3^{\text {rd }}$ quartile] of the: CCs, RMSE and REs of the reconstructed potentials along the time.

\begin{tabular}{c|c|c|c}
\hline Method & Dataset & CCs & REs (L2-norm) \\
\hline \multirow{2}{*}{ CRESO } & SSPs & $0.8[0.75,0.9]$ & $0.57[0.41,0.68]$ \\
\cline { 2 - 4 } & SSWs & $0.61[0.60,0.83]$ & $0.24[0.15,0.38]$ \\
\hline \multirow{2}{*}{ L-curve } & SSPs & NA* & $0.61[0.4,1]$ \\
\cline { 2 - 4 } & SSWs & NA* & $1[0.4,1]$ \\
\hline \multirow{2}{*}{ U-curve } & SSPs & $0.83[0.77,0.9]$ & $0.44[0.28,0.54]$ \\
\cline { 2 - 4 } & SSWs & $0.72[0.68,0.84]$ & $0.26[0.11,0.37]$ \\
\hline \multirow{2}{*}{ ADPC } & SSPs & $0.84[0.81,0.86]$ & $0.35[0.23,0.46]$ \\
\cline { 2 - 4 } & SSWs & $0.78[0.42,0.84]$ & $0.25[0.11,0.37]$ \\
\hline
\end{tabular}

Table 2. Median [min, max] of the CCs and of the REs for the activation time (AT) maps. NA*: Not applicable because the computation of the ATs is inhibited due to the over-regularized solution provided by L-curve.

\section{Discussion and Conclusions}

We presented ADPC, a new method to choose the regularization parameter for Tikhonov regularization and we showed the feasibility of this new method and the existent U-curve method (never before used for ECGI problems). Our results showed the importance of the choice of the regularization parameter. 
For the in-silico data used here, we found that the L-curve provided an over-regularized solution for the single site pacing (SSP) in the RV and for three of the single spiral waves simulations (SSWs), inhibiting the computation of the AT map for these SSWs. In figure 2, for one of the SSWs, we can clearly see how the L-curve regularization parameter provided is far above the other regularization parameters, as well as of the moment where the SVs start to decay to zero faster than the respective right hand side coefficients. For the cases that this happens, a few SVs are highly weighted in the Tikhonov solution (3), and this results in a highly over-regularized solution. In figure 3 (above), we can visualize this fact in terms of the reconstructed potentials in a random epicardial point along the time (for a SSP in the RV); when using the L-curve regularization parameter. Furthermore, figure 3 (below) shows that ADPC improves also the reconstruction in a SSP in the $\mathrm{LV}$.

While CRESO seems to give lower RMSE in agreement with the work of Rudy [10] for the single site pacing simulations (table 1), the U-curve and specially the ADPC provide higher CCs in terms of potentials (in time) and lower REs. Furthermore, the CCs of the ATs are also higher for the U-curve and the ADPC, especially for the SSWs (5-11\% of improvement), while the REs are not affected for the SSWs and decreased for the SSPs in a 21\% respect to the usual CRESO method (table 2).

We would like to remark that this study provides results for the ECGI MFS problem, such as described in $[9,10]$. However, it is well known that a suitable automatic regularization parameter choice method depends on the inverse problem treated [12]. Therefore, if the ECGI problem is numerically approximated by a different method such as the finite element method or the boundary element method, this study must be repeated for the specific problem in each case. DPC provides an interval that any Tikhonov regularization parameter should fulfill. The empirical choice of the lower threshold for the ADPC algorithm is working well for the ECGI MFS problem [9,10] where the decay behavior of SVs does not have jumps and always decays slowly for the first SVs $i \sim N T$, $i \leq N T$, and the respective coefficients of the solution start to be unstable exactly at that moment. The empirical lower threshold of DPC (step 3), chosen in our ADPC algorithm, may be differently adapted for others approaches with different SV decayment behavior. More detail of DPC can be found in earlier work $[12,18]$ that may help to the reader to adapt it to a specific inverse problem. Equally in different inverse problems involving spatio-temporal solutions, the choice of the mode or mean instead of median could be more accurate for step 4 than the median proposed here. Nevertheless, we believe that, as shown for our specific problem, a good adaptation of ADPC for each different numerical approach of ECGI or other ill-posed problems should provide suitable results since ADPC is really focused on the mathematical DPC that any Tikhonov solution should fulfill.

Finally, it seems advisable to test the four regularization parameter choice methods with experimental data with different pathologies and study the different solutions in future work.

Acknowledgements. This study received financial support from the French Government under the "Investments of the Future" program managed by the National Research 
Agency (ANR), Grant reference ANR-10-IAHU-04 and from the Conseil Régional Aquitaine as part of the project "Assimilation de données en cancérologie et cardiologie". This work was granted access to the HPC resources of TGCC under the allocation x2016037379 made by GENCI.

\section{References}

1. Shah, A. (2015). Frontiers in Noninvasive Cardiac Mapping, an Issue of Cardiac Electrophysiology Clinics. Elsevier Health Sciences, 7 (1).

2. Rudy Y. (2013). Noninvasive electrocardiographic imaging of arrhytmogenic substrates in humans. Circulation research, 112, 849-862.

3. Wang, Y., et al. (2011). Noninvasive electro anatomic mapping of human ventricular arrhythmias with electrocardiographic imaging. Science translational medicine, 3(98), 98ra8498ra84.

4. Ramanathan, C., et al. (2004). Noninvasive electrocardiographic imaging for cardiac electrophysiology and arrhythmia. Nature medicine, 10(4), 422-428.

5. Dubois R., et al. (2015). Non-invasive cardiac mapping in clinical practice: Application to the ablation of cardiac arrhythmias. Journal of Electrocardiology, 48(6), 966-974.

6. Haissaguerre M, et al. (2013). Noninvasive panoramic mapping of human atrial fibrillation mechanisms: a feasibility report. Journal of Cardiovascular Electrophysiology, 24, 711-717.

7. Cochet, H., et al. (2014). Cardiac arrythmias: multimodal assessment integrating body surface ECG mapping into cardiac imaging. Radiology, 271(1), 239-247.

8. Cluitmans, M. J. M., et al. (2015). Noninvasive reconstruction of cardiac electrical activity: update on current methods, applications and challenges. Netherlands Heart Journal, 23(6), 301-311.

9. Wang Y., \& Rudy Y. (2006). Application of the method of fundamental solutions to potentialbased inverse electrocardiography. Annals of Biomedical Engineering, 34, 1272-88.

10. Rudy, Y. (2004). U.S. Patent No. 6,772,004. Washington, DC: U.S. Patent and Trademark Office.

11. Milanič, M., et al. (2014). Assessment of regularization techniques for electrocardiographic imaging. Journal of electrocardiology, 47(1), 20-28.

12. Hansen, P. C. (2010). Discrete inverse problems: insight and algorithms (Vol. 7). SIAM.

13. Tsai, C. C., et al. (2006). Investigations on the accuracy and condition number for the method of fundamental solutions. Computer Modeling in Engineering and Sciences, 16(2), 103.

14. Colli-Franzone, P., et al. (1985). A mathematical procedure for solving the inverse potential problem of electrocardiography. Analysis of the time-space accuracy from in vitro experimental data. Mathematical Biosciences, 77(1-2), 353-396.

15. Ruan, S., Wolkowicz, G. S. K., \& Wu, J. (Eds.). (1999). Differential equations with applications to biology (Vol. 21). American Mathematical Soc.

16. Hansen, P. C., \& O'Leary, D. P. (1993). The use of the L-curve in the regularization of discrete ill-posed problems. SIAM Journal on Scientific Computing, 14(6), 1487-1503.

17. Krawczyk-Stańdo, D., \& Rudnicki, M. (2007). Regularization parameter selection in discrete ill-posed problems - the use of the U-curve. International Journal of Applied Mathematics and Computer Science, 17(2), 157-164.

18. Chamorro-Servent, J., et al. (2011). Feasibility of U-curve method to select the regularization parameter for fluorescence diffuse optical tomography in phantom and small animal studies. Optics express, 19(12), 11490-11506. 
19. Duchateau, J., Potse, M., \& Dubois, R. (2016). Spatially Coherent Activation Maps for Electrocardiographic Imaging. IEEE Transactions on Biomedical Engineering, (in print).

20. Ten Tusscher, K. H. W. J., et al. (2004). A model for human ventricular tissue. American Journal of Physiology-Heart and Circulatory Physiology, 286(4), H1573-H1589.

21. Potse, M. et al. (2009). Cardiac anisotropy in boundary-element models for the electrocardiogram. Medical and Biological Engineering and Computing, 47(7), 719-729.

22. Hansen, P. C., Regularization Tools Version 4.0 for Matlab 7.3, Numerical Algorithms, 46 (2007), pp. 189-194.

23. Ghodrati, A., et al. (2006). Wavefront-based models for inverse electrocardiography. IEEE transactions on biomedical engineering, 53(9), 1821-1831.

24. Chamorro-Servent, J., et al. (2016, September). Adaptive placement of the pseudo-boundaries improves the conditioning of the inverse problem. In Computing in Cardiology, 43, 705-708. 